\title{
Morphological Shape features for Classification of Textures based on Fuzzy Texture Element
}

\author{
M. Rama Bai \\ Associate Professor \\ M.G.I.T, JNTUH, Hyderabad \\ Andhra Pradesh, India
}

\author{
Dr.V.Venkata Krishna \\ Principal \\ C.I.E.T, JNTUK, Kakinada \\ Rajahmundry, Andhra Pradesh, India
}

\author{
J.Sasi Kiran \\ Associate Professor \\ V.V.I.T, JNTUH, Hyderabad \\ Andhra Pradesh, India
}

\begin{abstract}
Texture is an important spatial feature useful for identifying objects or regions of interest in an image. The present paper derives a new set of texture features, which are morphological shape components derived from the fuzzy texture elements of a $3 \times 3$ mask. The proposed fuzzy texture element patterns (FTP's) extract textural information of an image with a more complete respect of texture characteristics in all the eight directions instead of only one displacement vector. The proposed FTP's retains discriminating power of texture elements. In the present paper, five simple morphological shape components are evaluated on each of the derived FTP. The experimental results on the five groups of texture images clearly show the efficacy and simplicity of the present method.
\end{abstract}

Keywords: Morphological Shape components, Textural information, Classification, Fuzzy texture element.

\section{INTRODUCTION}

The present paper used textural properties for classification of textures $[1,2,3,4,5]$. Texture is the term used to characterize the surface of a given object or phenomenon and is undoubtedly one of the main features used in image processing, pattern recognition and multispectral scanner images obtained from aircraft or satellite platforms to microscopic images of cell cultures or tissue samples. That's why the research on texture classification and analysis has received considerable attention in recent years. There are several other areas like metallography [6] and umber processing [7] that make extensive use of textural features such as grain patterns and shapes, size, and distribution for classifying and analyzing specimens.

The study of patterns on textures is recognized as an important step in characterization and recognition of texture $[17,18,24$, $25,26]$. Various approaches are in use to investigate the textural and spatial structural characteristics of image data, including measures of texture [8], Fourier analysis [9, 10], fractal dimension [11], variograms [12,13] and local variance measures [14]. Fourier analysis is found as the most useful when dealing with regular patterns within image data. It is used to filter out speckle in radar data [15] and to remove the effects of regular agricultural patterns in image data [15]. Study of regular patterns based on fundamentals of local variance was carried out recently [16].

Texture and pattern were recognized as important attributes of image data. Patterns are used extensively in the visual interpretation of image data, in which texture is often more important than the other image attributes. Depending on the context, the word pattern has many different interpretations.

Textures are classified by pattern based approaches $[11,19,20$, 15]. This explains that the texture is characterized not only by gray value at a given pixel, but also by the gray value pattern in the surrounding pixels. The texture has both local and global meaning, in the sense that it is characterized by the invariance of certain local attributes that are distributed over a region of an image.

Based on this texture and pattern relation the present paper attempted to classify various texture images based on fuzzy texture element patterns (FTP's), which is different from the earlier studies. The proposed FTP method retains discriminating power of texture elements derived by He and Wang [21, 22] in a better way.

The present paper is organized as follows. The section 2 defines morphology and shape features, Section 3 describe the methodology, the results and discussions are presented in section 4 and conclusions are listed in section 5 .

\section{MORPHOLOGY \& SHAPE FEATURES}

Mathematical morphology is a well-founded non-linear theory of image processing [27, 28, 29, 30, 31]. Its geometry-oriented nature provides an efficient framework for analyzing object shape characteristics such as size and connectivity, which are not easily accessed by linear approaches. Morphological operations take into consideration the geometrical shape of the image objects to be analyzed. Mathematical morphology is theoretically founded on set theory. It contributes a wide range of operators to image processing, based on a few simple mathematical concepts. An image can be represented by a set of pixels. A morphological operation uses two sets of pixels, i.e., two images: the original data image to be analyzed and a structuring element (also called kernel) which is a set of pixels or a pattern constituting a specific shape such as a line, a disk, or a square. A structuring element is characterized by a welldefined shape (such as line, segment, or any pattern), size, and origin. Its shape can be regarded as a parameter to a morphological operation. In mathematical morphology, neighborhoods are, defined by the structuring element, i.e., the shape of the structuring element determines the shape of the neighborhood in the image.

Mathematical Morphology is based on logical transformations of the image (this is no constraint when these transformations are generalized in terms of set definitions) carried out by using the set theoretical operations. This would enable us to make several measurements on the image, like trend, directional effect 
and holes. The basic step in morphology is to compare the objects which are to be analyzed with an object of known shape, termed structuring element (This forms one mode of definition). The result of comparison of an object under study (analogous to universe) with a structuring element (analogous to a defined set) causes an image transformation.

\section{METHODOLOGY}

In a square-raster digital image, each pixel is surrounded by eight neighboring pixels. The local texture information for a pixel can be extracted from a neighborhood of $3 \times 3$ pixels, which represents the smallest complete unit having eight directions surrounding the pixel. In the present paper texture features on a $3 \times 3$ mask are evaluated based on the central pixel. A neighborhood of $3 \times 3$ pixels is denoted by a set containing nine elements: $\mathrm{P}=\{\mathrm{P} 0, \mathrm{P} 1 \ldots \mathrm{P} 8\}$, here $\mathrm{P} 0$ represents the intensity value of the central pixel and $\mathrm{Pi}\{\mathrm{i}=1,2 \ldots 8\}$, is the intensity value of the neighboring pixel $\mathrm{i}$ as shown in Fig.1.

\begin{tabular}{|l|l|l|}
\hline$P_{1}$ & $P_{2}$ & $P_{3}$ \\
\hline$P_{8}$ & $P_{0}$ & $P_{4}$ \\
\hline$P_{7}$ & $P_{6}$ & $P_{5}$ \\
\hline
\end{tabular}

Fig 1: Representation of a $3 \times 3$ neighborhood

The present paper labels eight neighbors of a $3 \times 3$ mask using five possible fuzzy patterns or values $\{0,1,2,3$ and 4$\}$ derived from the fuzzy code as depicted in Equation 1 instead of ternary or binary values. In natural images, due to the presence of noise and the different processes of capture and digitations, even if the human eye perceives two neighboring pixels as equal, they rarely have exactly the same intensity value. To avoid this imprecision and be able to represent the vagueness within the processes, the present paper make use of fuzzy logic and fuzzy techniques in deriving shape elements. Therefore to deal classification effect by morphological shape components, with regions of natural images perceived as homogeneous by human beings, the present paper proposes a fuzzy texture pattern encoding. Unlike the ternary texture element, wherein each morphological shape component has one of three possible values $(0,1$, or 2$)$, in the FTP five values are assigned, each showing the degree to which the grey levels of surrounding pixels are lighter, similar, or darker than that of the seed pixel as shown in Fig 2.

$$
P=\left\{\begin{array}{lll}
0 & \text { if } P_{i}<P_{0} \text { and } P_{i}<x \\
1 & \text { if } \quad P_{i}<P_{0} \text { and } P_{i}>P_{x} \\
2 & \text { if } & P_{i}=P_{0} \\
3 & \text { if } \quad P_{i}>P_{0} \text { and } P_{i}>y \\
4 & \text { if } \quad P_{i}>P_{0} \text { and } P_{i}<y
\end{array}\right\} \text { for } \mathrm{i}=1,2, \ldots, 8
$$

Where $\mathrm{P}$ is the obtained fuzzy code, $\mathrm{Pi}$ is the original pixel value at position i. P0 is the central pixel value, and $\mathrm{x}$ and $\mathrm{y}$ are user specified lag values. A $3 \times 3$ neighborhood that is labeled by fuzzy code is called as FTP. A pattern or a mask can be represented by a morphological structuring element. The Figs.

2(a) and 2(b) show the gray level values of a $3 \times 3$ neighborhood and the corresponding FTP.

\begin{tabular}{|l|l|l|}
\hline 63 & 28 & 40 \\
\hline 88 & 40 & 35 \\
\hline 67 & 40 & 21 \\
\hline
\end{tabular}

(a)

\begin{tabular}{|l|l|l|}
\hline 4 & 0 & 2 \\
\hline 4 & & 1 \\
\hline 4 & 2 & 0 \\
\hline
\end{tabular}

(b)

\section{Fig 2(a) : Sample Gray level Neighborhood 2(b): Fuzzy labeling of texture element.}

On the FTP representation of a $3 \times 3$ mask the present study evaluated fuzzy texture features which represents the morphological shape component. The present paper derived five different morphological shape components named as Diamond Fuzzy Texture element Pattern(DFTP-I), Corner Fuzzy Texture element Pattern(CFTP-I), Left Fuzzy Texture element Pattern(LFTP-I), Right Fuzzy Texture element Pattern(RFTP-I) and Blob Fuzzy Texture element Pattern(BFTP-I), where 'I' ranges from 0 to 4 . That is, DFTP-0 means the diamond shape component formed with FTP values 0. Each morphological shape components can be represented in 5-ways by using the FTP which is shown in Fig 3.

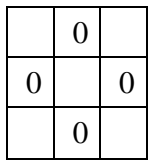

(i)

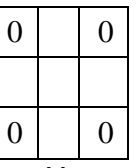

(i)

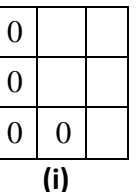

(i)

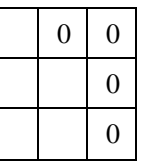

(i)

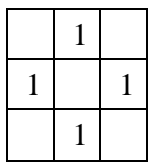

(ii)

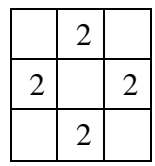

(iii)

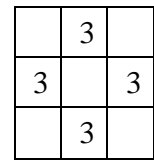

(iv)

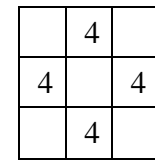

(v)
Fig 3: (a) Representation of DFTP-I.
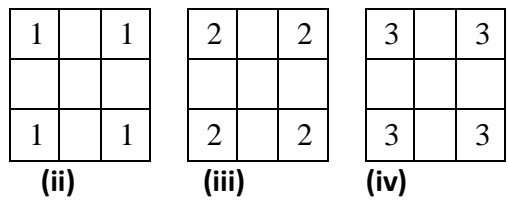

(iv)

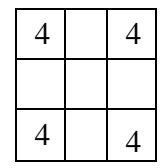

(v)

Fig 3: (b) Representation of CFTP-I.
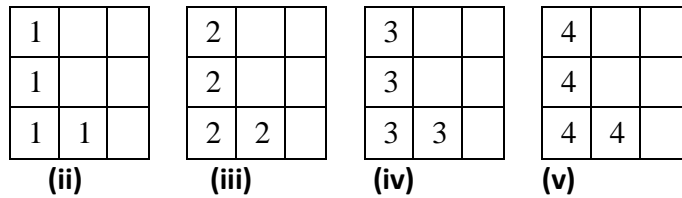

(v)

Fig 3: (c) Representation of LFTP-I.

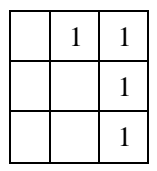

(ii)

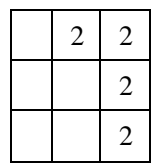

(iii)

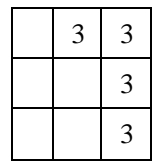

(iv)

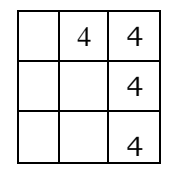

(v)
Fig 3: (d) Representation of RFTP-I. 


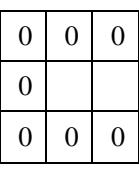

(i)

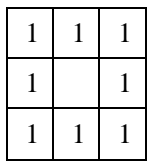

(ii)

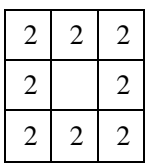

(iii)

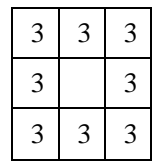

(iv)

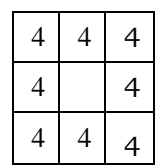

(v)
Fig 3: (e) Representation of BFTP-I.

For the classification of textures the frequency of occurrence of each morphological shape component with different FTP is counted using the Algorithm 1. One can enumerate large number of morphological shape components using FTP 0, 1, 2, 3 and 4. The novelty of the present work is, it uses only five different types of morphological shape components on FTP's.

Algorithm 1: Classification of textures based on morphological shape components with different FTP.

\section{Begin}

1. Read the original texture images $T_{k}$, where $k=1$ to $n$ with dimension $\mathrm{N} \times \mathrm{M}$.

2. Convert each $3 \times 3$ mask of the gray level texture image into a FTP.

The gray level texture image $T_{k}$ is assigned a fuzzy value $0,1,2,3$ or 4 in the following way

$$
\begin{aligned}
& \mathrm{CP}=\mathrm{T}_{\mathrm{k}}(2,2) \\
& \mathrm{Y}=\mathrm{CP}+10 \text {; } \\
& \mathrm{X}=\mathrm{CP}-10 \text {; } \\
& \text { for } u=1: 3 \\
& \text { for } \mathrm{v}=1: 3 \\
& \text { if } \mathrm{T}_{\mathrm{k}}(\mathrm{u}, \mathrm{v})<\mathrm{CP} \& \& \mathrm{~T}_{\mathrm{k}}(\mathrm{u}, \mathrm{v})<\mathrm{X} \\
& \operatorname{img}(\mathrm{u}, \mathrm{v})=0 \\
& \text { else } \\
& \mathrm{T}_{\mathrm{k}}(\mathrm{u}, \mathrm{v})=1 \\
& \text { end } \\
& \text { if } \mathrm{T}_{\mathrm{k}}(\mathrm{u}, \mathrm{v})==\mathrm{CP} \\
& \operatorname{img}(\mathrm{u}, \mathrm{v})=2 \\
& \text { end } \\
& \text { if } \mathrm{T}_{\mathrm{k}}(\mathrm{u}, \mathrm{v})>\mathrm{CP} \& \& \mathrm{~T}_{\mathrm{k}}(\mathrm{u}, \mathrm{v})>\mathrm{Y} \\
& \operatorname{img}(\mathrm{u}, \mathrm{v})=4 \\
& \text { else } \\
& \operatorname{img}(\mathrm{u}, \mathrm{v})=3 \\
& \text { end } \\
& \text { end }
\end{aligned}
$$

3. Represent the given shape patterns $\mathrm{P}_{\mathrm{ij}}$ on $3 \times 3$ mask elements, where $\mathrm{i}=1$ to 5 are morphological shape components and $\mathrm{j}=1$ to 5 represent FTP's $0,1,2,3$ and 4 .

4. Compute frequency occurrence $\left(\mathrm{FO}_{\mathrm{i}}\right)$ of each shape pattern $\mathrm{P}_{\mathrm{ij}}$.

5. Compute the number of occurrence of each shape pattern (NSP $\mathrm{Nij}_{\mathrm{ij}}, \mathrm{i}=1$ to 5 and $\mathrm{j}=1$ to 5 ) for each category of the texture $\mathrm{T}_{\mathrm{k}}$.

\section{end}

\section{RESULTS AND DISCUSSIONS}

To evaluate a good classification and recognition based on the morphological shape components of FTP, the present paper initially computed the frequency occurrences of each morphological shape component with each FTP. The novelty of the present scheme is, it classifies the given set of textures without any distance function, and that, it reduces the time complexity. For the classification purpose the present paper considers 5 groups of textures namely Brick, Fabric, Granite Marble and Mosaic each with six textures of 256 × 256 resolutions as shown in Fig. 4, 5, 6, 7 and 8 respectively. These texture image groups are collected mainly from the VisTex album and other standard albums. Based on Algorithm 1 the frequency of occurrence of each morphological shape component using different FTP for each group of textures is evaluated and represented in Tables 1, 2, 3, 4 and 5 for Brick, Fabric, Granite, Marble and Mosaic textures respectively.

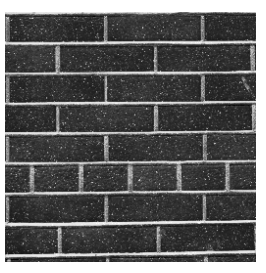

(a)

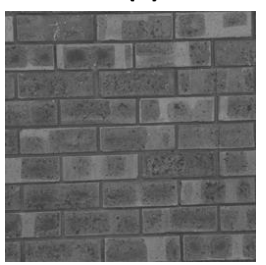

(d)

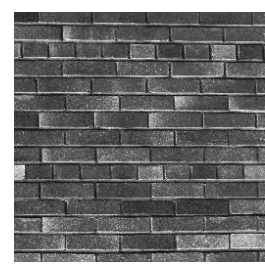

(b)

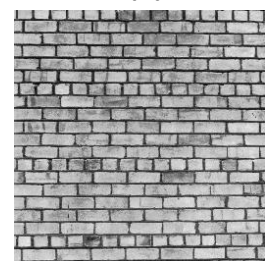

(e)

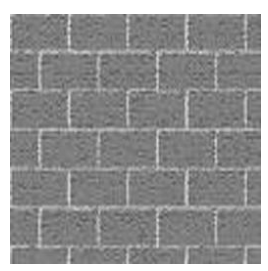

(c)

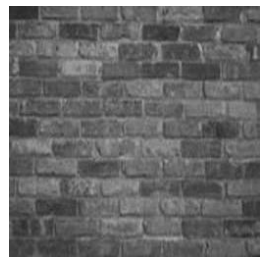

(f)
Fig 4: Original images of six Brick textures from (a)-(f).

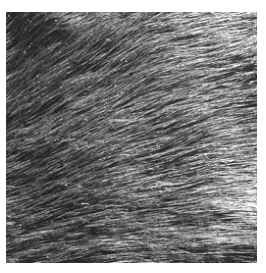

(a)

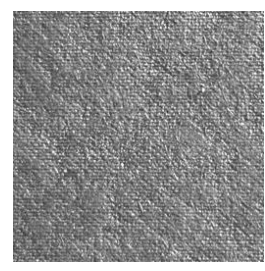

(b)

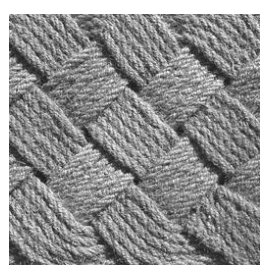

(c) 


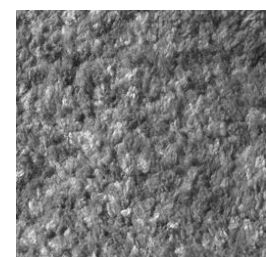

(d)

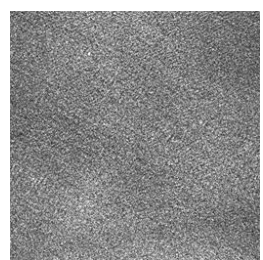

(e)

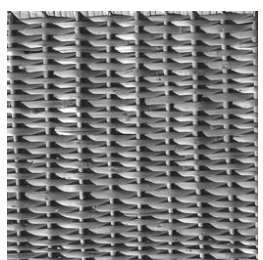

(f)
Fig 5: Original images of six Fabric textures from (a)-(f).

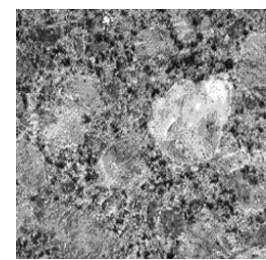

(a)

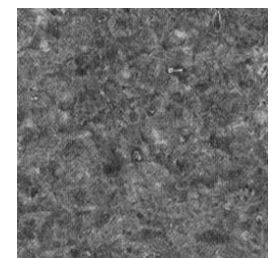

(d)

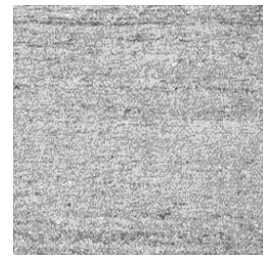

(b)

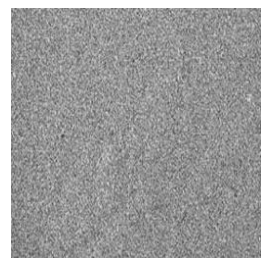

(e)

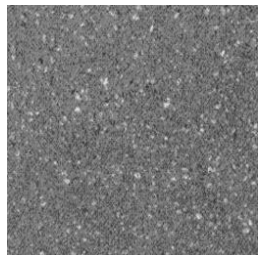

(c)

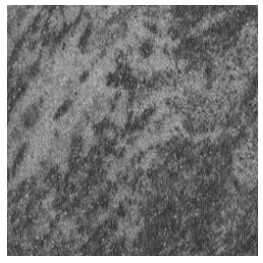

(f)
Fig 6: Original images of six Granite textures from (a)-(f).

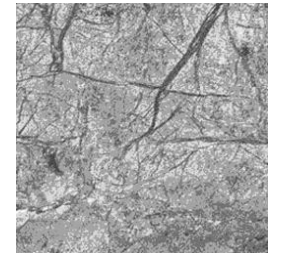

(a)

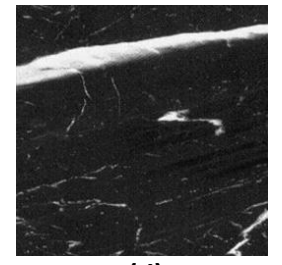

(d)

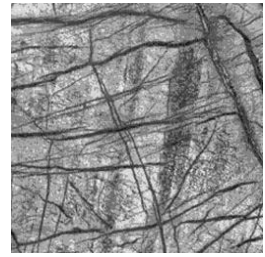

(b)

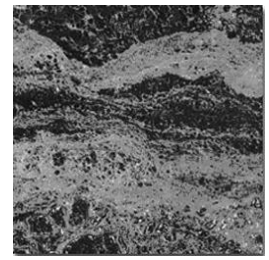

(e)

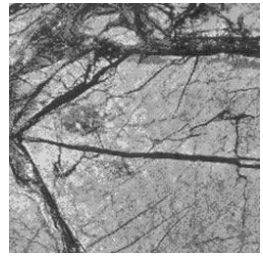

(c)

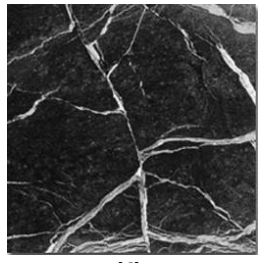

(f)
Fig 7: Original images of six Marble textures from (a)-(f).

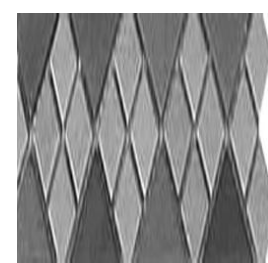

(a)

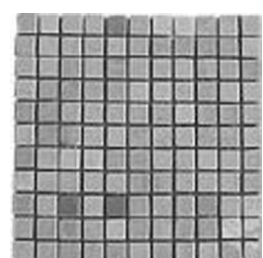

(b)

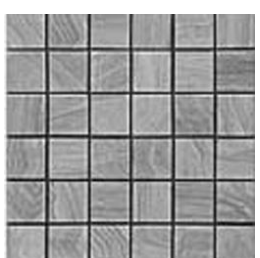

(c)

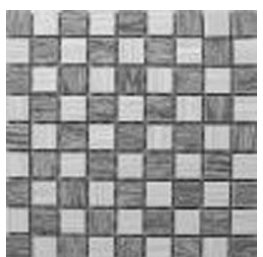

(d)

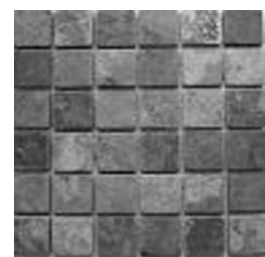

(e)

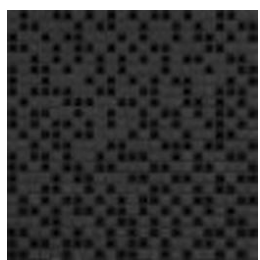

(f)
Fig 8: Original images of six Mosaic textures from (a)-(f).

The present study utilized one dimensional (1D) and two dimensional (2D) analysis or plots based on the frequency count of morphological shape components using FTP's for the classification purpose.

\subsection{Classification Based on 1D Survey of Morphological Shape Components Using FTP's}

From the frequency of occurrences of morphological shape components on FTP's of Tables 1 to 5, it is clearly evident that the Fabric and Granite textures can be classified easily based on the frequency of occurrences of DFTP-2 which results a zero count for these two groups of textures as shown in Table 2 and Table 5. It is also clearly evident that Mosaic textures can be classified as another group based on the frequency of occurrences of DFTP-4 which results a zero count for this group of texture. The classification using 1D survey based on DFTP-2 is shown in the form of flowchart in Fig.9. That is from the 1D survey of morphological shape components based on FTP's the following three classes are found from the considered five groups of textures.

Class-1: $\{$ Fabric, Granite $\}$

Class-2: $\{$ Mosaic $\}$

Class-3: $\{$ Brick, Marble $\}$

Further classification that is individual classification of Class-1 and Class-3 textures is not possible by the proposed 1D survey of morphological shape components using FTP's. From the above 1D survey it is clearly evident that only diamond shape components on FTP of a $3 \times 3$ mask results a good classification and all other four morphological shape components failed in classification of textures. The same morphological shape components on texture elements without fuzzy logic has resulted only two classification groups [23]. 
Table 1. Frequency of occurrence of each morphological shape component of Brick textures using FTP.

\begin{tabular}{|c|c|c|c|c|c|c|c|c|c|c|c|c|c|c|c|c|c|c|c|c|c|c|c|c|c|}
\hline \multirow{2}{*}{ Image } & \multicolumn{5}{|c|}{ Diamond } & \multicolumn{5}{|c|}{ Corners } & \multicolumn{5}{|c|}{ Left - L } & \multicolumn{5}{|c|}{ Right - L } & \multicolumn{5}{|c|}{ Blob } \\
\hline & $\mathbf{0}$ & 1 & 2 & 3 & 4 & $\mathbf{0}$ & 1 & 2 & 3 & 4 & 0 & 1 & 2 & 3 & 4 & $\mathbf{0}$ & 1 & 2 & 3 & 4 & 0 & 1 & 2 & 3 & 4 \\
\hline & 73 & 1 & t & 04 & 52 & 1 & 81 & 2 & $8 \delta$ & 219 & 556 & 22 & 1 & 11 & 128 & 565 & 120 & 0 & 99 & 135 & 333 & 0 & 0 & 1 & 17 \\
\hline Brick2 & 21 & 64 & 9 & 71 & 141 & 85 & 56 & 1 & 41 & 422 & 554 & 71 & 1 & 47 & 224 & 513 & 03 & 1 & 46 & 212 & 249 & 3 & 0 & 2 & 50 \\
\hline Brick3 & 0 & 101 & 2 & 4 & 40 & 14 & 170 & 6 & 43 & 7 & 32 & 330 & 23 & 303 & 41 & 63 & 361 & 12 & 278 & 52 & 0 & 30 & 3 & 38 & 0 \\
\hline D & 6 & 227 & 8 & 71 & 16 & 91 & 227 & 2 & 230 & 80 & 40 & 345 & 11 & 355 & 53 & 43 & 324 & 16 & 334 & 59 & 0 & 44 & 0 & 53 & 14 \\
\hline & 97 & 88 & 6 & 7 & 121 & 232 & 77 & 1 & 46 & 542 & 287 & 116 & 1 & 69 & 312 & 261 & 9 & 3 & 89 & 283 & 48 & 8 & 0 & 9 & 74 \\
\hline ick6 & 0 & 143 & 32 & 19 & 32 & 12 & 205 & 22 & 181 & 11 & 11 & 339 & 45 & 304 & 9 & 15 & 394 & 45 & 328 & $\mathrm{c}$ & 0 & 46 & 5 & 31 & 0 \\
\hline erag & 66 & 123 & 12 & 01 & \begin{tabular}{|l|}
67 \\
\end{tabular} & 299 & 136 & 6 & 122 & 214 & 247 & 221 & 14 & 198 & 128 & 243 & 228 & 3 & 96 & 125 & 06 & 23 & 1 & 22 & 26 \\
\hline
\end{tabular}

Table 2. Frequency of occurrence of each morphological shape component of Fabric textures using FTP.

\begin{tabular}{|c|c|c|c|c|c|c|c|c|c|c|c|c|c|c|c|c|c|c|c|c|c|c|c|c|c|}
\hline \multirow{2}{*}{ Image } & \multicolumn{5}{|c|}{ Diamond } & \multicolumn{5}{|c|}{ Corners } & \multicolumn{5}{|c|}{ Left - L } & \multicolumn{5}{|c|}{ Right - L } & \multicolumn{5}{|c|}{ Blob } \\
\hline & 0 & 1 & 2 & 3 & 4 & $\mathbf{0}$ & 1 & 2 & 3 & 4 & 0 & 1 & 2 & 3 & 4 & $\mathbf{0}$ & 1 & 2 & 3 & 4 & $\mathbf{0}$ & 1 & 2 & 3 & 4 \\
\hline Fabric1 & 395 & 12 & 0 & 12 & 257 & 835 & 7 & 0 & 7 & 737 & 655 & 15 & 0 & 12 & 460 & 592 & 12 & 0 & 8 & 480 & 220 & 2 & 0 & 1 & 119 \\
\hline Fabric2 & 380 & 5 & 0 & 6 & 181 & 739 & 1 & 0 & 0 & 554 & 775 & 8 & 0 & 0 & 525 & 785 & 2 & 0 & 3 & 540 & 285 & 0 & 0 & 0 & 110 \\
\hline Fabric3 & 469 & 9 & 0 & 7 & 370 & 598 & 3 & 0 & 2 & 549 & 732 & 6 & 0 & 8 & 596 & 668 & 12 & 0 & 2 & 613 & 218 & 0 & 0 & 0 & 206 \\
\hline Fabric4 & 134 & 8 & 0 & 11 & 87 & 318 & 15 & 0 & 5 & 174 & 501 & 28 & 0 & 30 & 394 & 540 & 50 & 0 & 22 & 407 & 71 & 0 & 0 & 0 & 28 \\
\hline Fabric5 & 575 & 2 & 0 & 2 & 517 & 791 & 3 & 0 & 1 & 651 & 849 & 4 & 0 & 0 & 719 & 808 & 7 & 0 & 0 & 742 & 340 & 0 & 0 & 0 & 270 \\
\hline Fabric6 & 222 & 11 & 0 & 8 & 84 & 1698 & 56 & 0 & 8 & 1398 & 808 & 115 & 0 & 16 & 475 & 545 & 76 & 0 & 6 & 686 & 79 & 4 & 0 & 2 & 41 \\
\hline Average & 363 & 8 & 0 & 8 & 249 & 830 & 14 & 0 & 4 & 677 & 720 & 29 & 0 & 11 & 528 & 656 & 27 & 0 & 7 & 578 & 202 & 1 & 0 & 1 & 129 \\
\hline
\end{tabular}

Table 3. Frequency of occurrence of each morphological shape component of Marble textures using FTP.

\begin{tabular}{|c|c|c|c|c|c|c|c|c|c|c|c|c|c|c|c|c|c|c|c|c|c|c|c|c|c|}
\hline \multirow{2}{*}{ Image } & \multicolumn{5}{|c|}{ Diamond } & \multicolumn{5}{|c|}{ Corners } & \multicolumn{5}{|c|}{ Left - L } & \multicolumn{5}{|c|}{ Right - L } & \multicolumn{5}{|c|}{ Blob } \\
\hline & $\mathbf{0}$ & 1 & 2 & 3 & 4 & $\mathbf{0}$ & 1 & 2 & 3 & 4 & $\mathbf{0}$ & 1 & 2 & 3 & 4 & 0 & 1 & 2 & 3 & 4 & 0 & 1 & 2 & 3 & 4 \\
\hline $\mathrm{Ma}$ & 155 & 93 & 5 & 73 & 123 & 336 & 46 & 1 & 40 & 340 & 494 & 102 & 1 & 75 & 478 & 487 & 97 & 3 & 89 & 459 & 87 & 6 & 0 & 8 & 103 \\
\hline Marble2 & 120 & 107 & 4 & 91 & 87 & 278 & 16 & 1 & 14 & 353 & 427 & 59 & 1 & 43 & 389 & 399 & 43 & 4 & 46 & \begin{tabular}{|l|}
409 \\
\end{tabular} & 75 & 1 & 0 & 0 & 81 \\
\hline Marble3 & \begin{tabular}{|l|}
97 \\
\end{tabular} & 76 & 5 & 97 & 92 & 233 & 37 & 3 & 32 & 251 & 352 & 82 & 7 & 69 & 350 & 361 & 106 & 6 & 91 & 347 & 58 & 2 & 2 & 5 & 60 \\
\hline Marble4 & 20 & 300 & 4 & 323 & 5 & 75 & 326 & 1 & 294 & 17 & 82 & 491 & 1 & 387 & 33 & 94 & 437 & 3 & 429 & 30 & 14 & 80 & 0 & 67 & 3 \\
\hline Marble5 & 150 & 89 & 3 & 75 & 111 & 314 & 33 & 0 & 18 & 289 & 459 & 63 & 0 & 50 & 419 & 453 & 62 & 0 & 46 & 416 & 99 & 9 & 0 & 3 & 79 \\
\hline Marble6 & 51 & 159 & 7 & 172 & 16 & \begin{tabular}{|l|}
139 \\
\end{tabular} & 178 & 1 & 153 & 66 & 194 & 303 & 2 & 261 & 109 & 179 & 328 & 7 & 257 & 99 & 30 & 48 & 0 & 43 & 5 \\
\hline Average & 99 & 137 & 5 & 139 & 72 & 229 & 106 & 1 & 92 & 219 & 335 & 183 & 2 & 148 & 296 & 329 & 179 & 4 & 160 & 293 & 61 & 24 & 0 & 21 & 55 \\
\hline
\end{tabular}

Table 4. Frequency of occurrence of each morphological shape component of Mosaic textures using FTP.

\begin{tabular}{|c|c|c|c|c|c|c|c|c|c|c|c|c|c|c|c|c|c|c|c|c|c|c|c|c|c|}
\hline \multirow{2}{*}{ Image } & \multicolumn{5}{|c|}{ Diamond } & \multicolumn{5}{|c|}{ Corners } & \multicolumn{5}{|c|}{ Left - L } & \multicolumn{5}{|c|}{ Right - L } & \multicolumn{5}{|c|}{ Blob } \\
\hline & $\mathbf{0}$ & 1 & 2 & 3 & 4 & $\mathbf{0}$ & 1 & 2 & 3 & 4 & $\mathbf{0}$ & 1 & 2 & 3 & 4 & $\mathbf{0}$ & 1 & 2 & 3 & 4 & $\mathbf{0}$ & 1 & 2 & 3 & 4 \\
\hline Mosaic1 & 0 & 6 & 38 & 2 & 0 & 175 & 423 & 31 & 427 & 165 & 48 & 515 & 84 & 417 & 91 & 86 & 425 & 96 & 480 & 35 & 0 & 3 & 24 & 1 & $\overline{0}$ \\
\hline Mosaic2 & 2 & 40 & 2 & 53 & 0 & 132 & 112 & 0 & 123 & 206 & 89 & 244 & 3 & 293 & 79 & 109 & 298 & 2 & 268 & 61 & 2 & 20 & 0 & 25 & 0 \\
\hline Mosaic3 & 0 & 60 & 4 & 64 & 0 & 120 & 182 & 1 & 192 & 91 & 38 & 350 & 13 & 381 & 38 & 40 & 422 & 9 & 363 & 17 & 0 & 30 & 0 & 39 & 0 \\
\hline Mosaic4 & 0 & 55 & 2 & 45 & 0 & 8 & 192 & 2 & 150 & 60 & 26 & 307 & 11 & 315 & 25 & 37 & 338 & 6 & 260 & 18 & 0 & 26 & 0 & 24 & 0 \\
\hline Mosaic5 & 0 & 51 & 6 & 32 & 0 & 3 & 184 & 3 & 116 & 34 & 5 & 518 & 14 & 436 & 13 & 8 & 485 & 12 & 395 & 12 & 0 & 29 & 2 & 17 & 0 \\
\hline Mosaic6 & 0 & 23 & 10 & 23 & 0 & 0 & 165 & 5 & 96 & 0 & 0 & 429 & 33 & 564 & 0 & 0 & 625 & 22 & 394 & 0 & 0 & 18 & 3 & 20 & 0 \\
\hline Average & 0 & 39 & 10 & 37 & 0 & 73 & 210 & 7 & 184 & 93 & 34 & 394 & 26 & 401 & 41 & 47 & 432 & 25 & 360 & 24 & 0 & 21 & 5 & 21 & 0 \\
\hline
\end{tabular}


Table 5. Frequency of occurrence of each morphological shape component of Granite textures using FTP.

\begin{tabular}{|c|c|c|c|c|c|c|c|c|c|c|c|c|c|c|c|c|c|c|c|c|c|c|c|c|c|}
\hline \multirow{2}{*}{ Image } & \multicolumn{5}{|c|}{ Diamond } & \multicolumn{5}{|c|}{ Corners } & \multicolumn{5}{|c|}{ Left - L } & \multicolumn{5}{|c|}{ Right - L } & \multicolumn{5}{|c|}{ Blob } \\
\hline & 0 & 1 & 2 & 3 & 4 & 0 & 1 & 2 & 3 & 4 & $\mathbf{0}$ & 1 & 2 & 3 & 4 & 0 & 1 & 2 & 3 & 4 & 0 & 1 & 2 & 3 & 4 \\
\hline & 210 & 19 & & & & 1 & 20 & & 15 & 2 & 563 & 36 & & 32 & 69 & 77 & 1 & & 33 & 515 & 9 & 2 & & & 9 \\
\hline & 161 & 15 & 0 & 4 & 139 & 399 & 13 & & 1 & 02 & 73 & 22 & & 18 & 484 & 464 & 29 & U & 22 & 456 & 100 & 1 & 0 & 2 & 8 \\
\hline 3 & 102 & 40 & 0 & 22 & 49 & 274 & 29 & & 6 & 409 & 388 & 78 & & 48 & 470 & 341 & 76 & 2 & 54 & 446 & 55 & 0 & 0 & 0 & 35 \\
\hline 4 & 46 & 4 & 0 & 6 & 35 & 170 & 51 & & 77 & 122 & 240 & 107 & 1 & 35 & 177 & 247 & 126 & 0 & 67 & 212 & 30 & 6 & 0 & 4 & 22 \\
\hline & 181 & 24 & 0 & 1 & 4 & 470 & 17 & ( & 14 & 468 & 526 & 25 & 0 & 17 & 493 & 529 & 27 & 0 & 18 & 482 & 125 & 0 & 0 & 0 & 102 \\
\hline & 73 & 46 & 0 & 44 & 157 & 280 & 32 & 0 & 20 & 270 & 354 & $10^{2}$ & 0 & 68 & 314 & 366 & 10 & 0 & 66 & 301 & 52 & 2 & 0 & 1 & 40 \\
\hline verage & 129 & 32 & 0 & 30 & 153 & 334 & 27 & 0 & 17 & 344 & 424 & 6 & & 45 & 18 & 21 & 67 & & 43 & 419 & 5 & & 0 & 2 & 74 \\
\hline
\end{tabular}

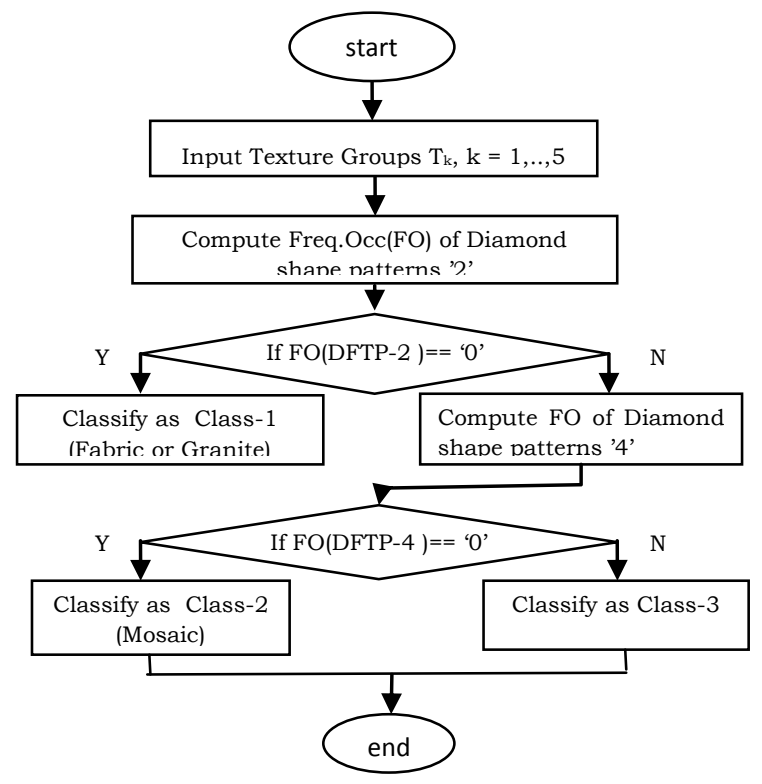

Figure 9. DFTP-2

\subsection{Classification of Textures Based on 2D Plots of Morphological Shape Components Using FTP's}

The present paper also classified the texture groups based on the $2 \mathrm{D}$ analysis on the morphological shape components using FTP's which are represented in the form of graphs. By this mechanism one can have one morphological shape component frequency count on $\mathrm{X}$-axis and the other on $\mathrm{Y}$-axis. There can be a total of 24 classification graphs that represent DFTP-0 on Xaxis and other morphological shape components or same morphological shape component with different FTP on Y-axis. By removing duplication the total number of such graphs for classification of textures based on the 2D analysis on the proposed five morphological shape components with FTP's 0,1 , 2, 3 and 4 is given by the Equation-2.

$$
T_{g}=n(n+1) / 2
$$

Where $\mathrm{T}_{\mathrm{g}}$ is total number of different graphs, $\mathrm{n}$ is $((\mathrm{S} \times \mathrm{P})-1)$, where $\mathrm{S}$ represents the total number of morphological shape components and P is the number of FTP's. This leads to an exhaustive study. To overcome this present approach plotted the following graphs as shown in Figures 10, 11, 12, 13 and 14 respectively. The graphs reveal the same factor that Fabric, Granite and Mosaic textures are plotted as different groups or in a region and other texture groups (Marble and Brick) are scattered entirely on the graphs. Thus a good classification system is built based on the 2D plots of morphological shape components using FTP for Mosaic, Fabric and Granite textures. From the 2D survey of morphological shape components based on FTP's the following four classes are found from the considered five groups of textures.

Class-1 :\{Mosaic $\}$

Class-2 : $\{$ Fabric $\}$

Class- $3:\{$ Granite $\}$

Class-4 :\{Brick and Marble $\}$

The above grouping clearly indicates the efficacy of the $2 \mathrm{D}$ graphs. However the present study suggests that it is not necessary to plot all graphs to classify textures. One can plot the frequency occurrence of DFTP-1 on X-axis and DFTP-4 on Yaxis, for a clear classification of Mosaic, Fabric and Granite textures as shown in Fig.10. The same classification of textures is also resulted in Fig.11, by plotting frequency occurrence of DFTP-3 on X-axis and DFTP-4 on Y-axis. Thus it reveals that a good classification can be resulted by diamond shape component feature based on FTP's on a $3 \times 3$ mask and one need not necessarily count the other four morphological shape components for classification of textures. Further classification that is individual classification of Brick and Marble textures is not possible by the proposed 2D surveys of morphological shape components using FTP's. Also it is to be noted that there is little bit of overlapping for Fabric and Granite textures using 2D graphs. However the similar 2D graphs without fuzzy coding using the same morphological shape components [23] resulted only two classification groups on the same texture groups.

Table 6. Percentage of occurrence of each morphological shape components with all patterns.

\begin{tabular}{|c|c|c|c|c|c|}
\hline \multirow{2}{*}{ Texture } & \multicolumn{5}{|c|}{ Percentage's of } \\
\cline { 2 - 6 } & DFTP & CFTP & LFTP & RFTP & BFTP \\
\hline Brick & 15.36 & 25.60 & 26.62 & 26.55 & 5.88 \\
\hline Fabric & 12.50 & 30.28 & 25.51 & 25.10 & 6.60 \\
\hline Marble & 12.68 & 20.65 & 30.74 & 30.77 & 5.15 \\
\hline Mosaic & 3.48 & 22.80 & 36.09 & 35.72 & 1.90 \\
\hline Granite & 10.84 & 23.15 & 30.42 & 30.45 & 5.12 \\
\hline Average & 10.97 & 24.50 & 29.88 & 29.72 & 4.93 \\
\hline
\end{tabular}


The present study also analyzed the percentage of occurrence factor of each morphological shape component with all patterns and it is represented in the Table 6 . The Table 6 reveals that CFTP, LFTP and RFTP morphological shape components have dominant grouping in all considered groups of texture images but fail in classification. The DFTP which is having moderate percentage, resulted as a good texture feature for classification. The BFTP shows the least percentage of morphological shape component and fails in classification.

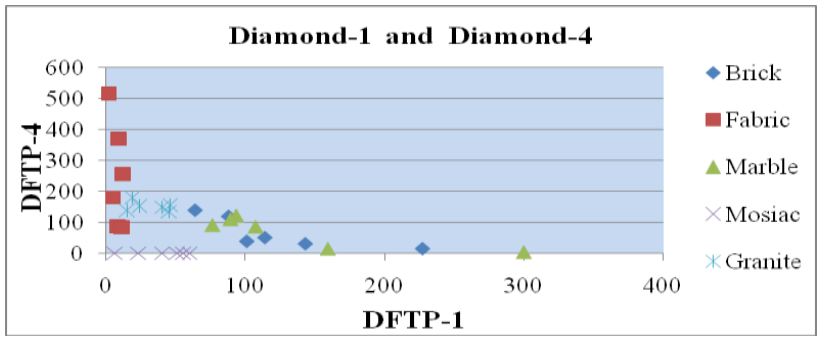

Fig 10: Graph between DFTP-1 and DFTP-4

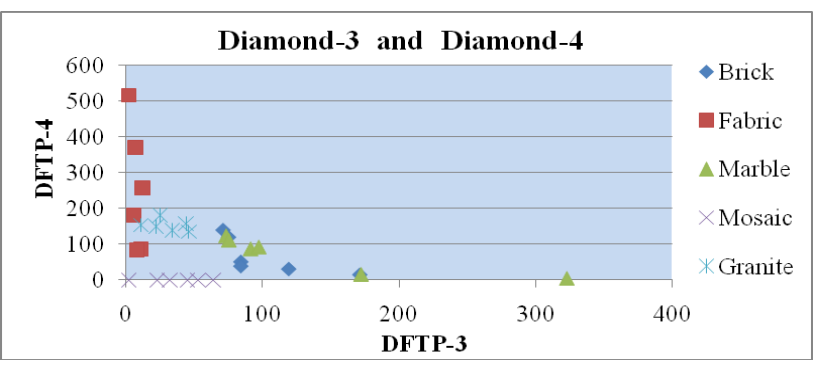

Fig 11: Graph between DFTP-3 and DFTP-4

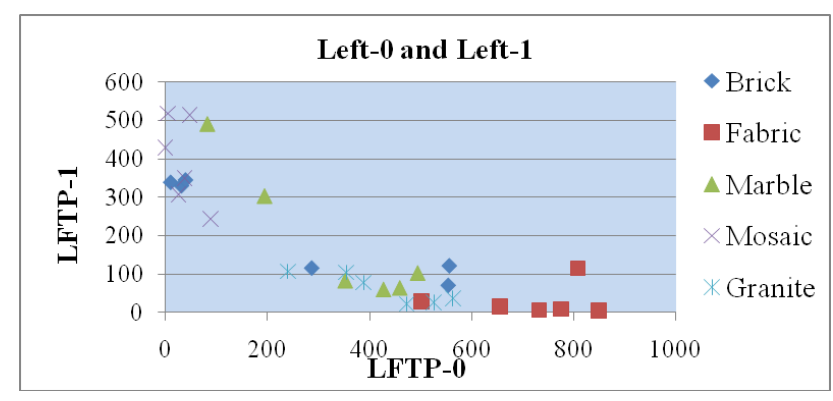

Fig 12: Graph between LFTP-0 and LFTP-1

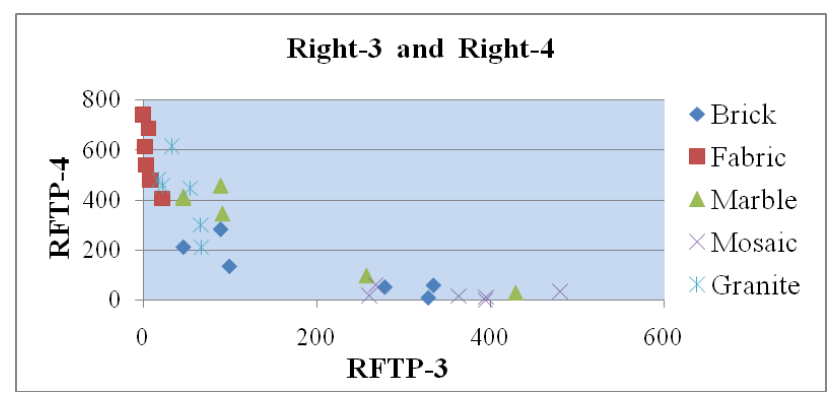

Fig 13: Graph between RFTP-3 and RFTP-4

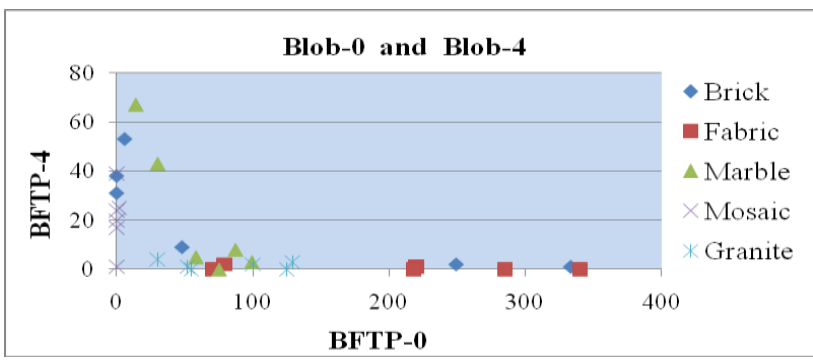

Fig 14: Graph between BFTP-0 and BFTP-4

\section{CONCLUSIONS}

The present study created a new direction for classification of textures based on morphological shape components derived from FTP of a 3x3 mask. By investigating texture classification using different morphological shape components the present paper concludes that diamond shape component contains more textural information for classification purpose than other morphological shape components. Based on the experimental results of $1 \mathrm{D}$ and $2 \mathrm{D}$ analysis the present paper concludes that one need not consider the other texture features CFTP, LFTP, RFTP and BFTP for classification purpose, since these morphological shape components contains least textural features. The FTP concept increased classification rate when compared to texture element patterns without fuzzy logic which classified the considered five groups of textures into only two groups of textures[23], resulting a poor classification. The present method proved as efficient tool when compared to previous one. The present paper also investigated that any stone texture contains more than $84 \%$ of occurrences of CFTP, LFTP and RFTP morphological shape components compared to DFTP with $11 \%$ and BFTP with $5 \%$ of occurrence.

\section{ACKNOWLEDGMENTS}

The authors would like to express their cordial thanks to Dr. K.V.V. Satya Narayana Raju, MLC and Chairman for providing facility to work at the advanced labs of Srinivasa Ramanujan Research Forum (SRRF)-GIET and Sri. K. Sasi Kiran Varma, Managing Director, Chaitanya Group of Institutions for providing moral support and encouragement towards research.

\section{REFERENCES}

[1] Caselles, V., Kimmel, R. and Sapiro, G., " Geodesic active contours, Int. J. Comput.Vis. ”,Vol.22, pp. 61-79, 1997.

[2] Eswara Reddy,B. and Vijaya Kumar,V.,'Texture Discrimination by Morphological Patterns after Edge Operator", International Conference (ICORG), R\&D Cell, JNT University, Hyderabad, 2006.

[3] Liu, X., Skidmore, A.K. and Oosten, H.V,'Integration of classification methods for improvement of land-cover map accuracy", ISPRS Journal of Photogrammetry and Remote Sensing, Vol.56, pp. 257-268, 2002. 
[4] Meisel, W.S.,"Computer Oriented Approaches to Pattern Recognition, Academic Press", New York, 1972.

[5] Tsai, D.M. and Wang, H.J.," Segmenting focused objects in complex visual images, Pattern Recognition", Letters. vol. 19, pp. 929 - 940, 1998.

[6] Richards, W. and Polit, A.," Texture Matching, Cybernetic", Vol 16, pp.155-162, 1974.

[7] Chen, G. and Yang, Y.H.H.," Edge Detection by Regularized Cubic B- spline Fitting”, IEEE Transactions on Systems, Man, and Cybernetics, Vol.25, pp.635-642, 1995.

[8] Lira, J. and Maletti, G," A supervised contextual classifier based on a region-growth algorithm", Computers and Geosciences, Vol.28, pp. 951-959, 2002.

[9] Jensen, J.R," Introduction to Digital Image Processing: A Remote Sensing Perspective", 2nd Edition. Princeton Hall, 1996.

[10] Tamura, H., Mori, S. and Yamawaki, Y.," Textural Features Corresponding to Visual Perception", IEEE Trans. on Systems, Man and Cybernetics, Vol.8, pp.460-473, 1978.

[11] Bhanu, B. and Peng, J.," Adaptive integrated image recognition and segmentation", IEEE Trans. Syst., Man, Cybern. C, Appl. Rev., Vol.30, pp. 427-441, 2000.

[12] Barandela, R. and Juarez, M.," Supervised classification of remotely sensed data with ongoing learning capability", International Journal of Remote Sensing, Vol.23, pp. 49654970, 2002.

[13] Morrone, M.C. and Owens, R.A.," Feature detection from local energy", Patterns Recognition Letters, Vol.6, pp. 303313, 1987.

[14] Sayre, K.M.," Recognition, A Study in the Philosophy of Artificial Intelligence", University of Notre Dame Press, Ohio, 1965.

[15] Heitger, F.," Feature Detection using Suppression and Enhancement. Technical Report TR-163", Communication Technology Laboratory, Swiss Federal Institute of Technology, 1995.

[16] Kashyap, R.L. and Khotanzad, A.," A Model based Method for Rotation Invariant Texture Classification", IEEE Trans. Pattern Anal. Machine Intell, Vol. 8, pp.472-481, 1986.

[17] Peder Klith Bocher and Keith R. McCloy," The Fundamentals of Average Local Variance: Detecting Regular Patterns", IEEE Trans. on Image Processing, Vol. 15, pp.300-310, 2006.

[18] Penaloza, M.A. and Welch, R.M," Feature selection for classification of polar regions using a fuzzy expert system", Remote Sensing of Environment, Vol.58, pp. 81-100, 1996.

[19] Biehl, L. and Landgrebe, D.," MultiSpec-a tool for multispectral-hyper spectral image data analysis. Computers and Geosciences", Vol.28, pp. 1153-1159, 2002.

[20] Binaghi, E., Madella, P., Montesano, M.G. and Rampini, A," Fuzzy contextual classification of multisource remote sensing images", IEEE Transactions on Geoscience and Remote Sensing, Vol.35, pp. 326-339, 1997.

[21] Dong-Chen He , Li Wang "Texture Feature Extraction from Texture Spectrum”, IEEE Trans, Vol.8,p.p 198719901990

[22] D.C.He and L.Wang, "Texture unit, texture spectrum andtexture analysis", in Proc of IGARSS'89,Vancouver,Canada, 1989,Vol.5,pp.2769-2771.

[23] V.Venkata Krishna, M.Rama Bai and V. Vijaya Kumar, "Extraction of shape components for classification of textures based on texture elements," IJCSNS, Vol.11,No.2, p.p 114-120, 2011.

[24] V. Vijaya Kumar, B. Eswara Reddy and U.S.N.Raju ,“A measure of patterns trends on various types of preprocessed images," IJCSNS, Vol.7 No.8, p.p. 253-257, 2007.

[25] V. Vijaya Kumar, B. Eswara Reddy , U.S.N.Raju and K. Chandra Sekharan "An Innovative Technique of Texture Classification and Comparison Based on Long Linear Patterns", Journal of Computer Science 3 (8):633$638,2007$.

[26] B. Eswara Reddy, A. Nagaraja Rao, A. Suresh and V.Vijaya Kumar "Texture Classification by simple patterns on edge direction movements", IJCSNS, Vol.7 No.11, p.p. 220-225, 2007.

[27] Dougherty E. and J. Astola," An Introduction to Nonlinear Image Processing", vol. TT 16, SPIE Optical Engineering Press, Washington, 1994

[28] Gasteratos," Mathematical morphology operations and structuring elements", In CVonline: On-Line Compendium of Computer Vision [Online]. R. Fisher(ed). Available: http://www.dai.ed.ac.uk/ CVonline/transf.htm, Dec. 2001, Section: Image Transformations and Filters.

[29] Maragos P., and Schafer R.W.," Morphological filters-part II, Their relations to median, order statistics, and stack filters", IEEE Trans., 1987, ASSP-35,(8), pp. 1170-1184.

[30] Maragos P., and Schafer R.W.," Morphological skeleton representation and coding of binary images", IEEE Trans. 1986, ASSP-34, pp. 1228-1244.

[31] Schonfeld D., and Goutsias J.,’Optimal morphological pattern restoration from noisy binary images", IEEE Trans. Pattern Anal. Mack Intell., 1991, 13, pp. 14-29.

\section{AUTHORS PROFILE}

M. Rama Bai received B.E (CSE) degree from Bharathiar University, Coimbatore (T.N), India in 1994. She worked as lecturer in Amrita Institute of Technology and Science, Coimbatore for three years and Sri Venkateshwara Institute of Science and Technology, Machilipatnam for two years. She joined as Assistant Professor in the Dept of Computer Science \& Engineering, Mahatma Gandhi Institute of Technology (MGIT) under JNT University, Hyderabad, India in 1999. She received her M.Tech (CSE) from College of Engineering, Osmania University, Hyderabad. At present she is working as Associate Professor in CSE Dept at MGIT, Hyderabad. She is pursuing her $\mathrm{Ph} . \mathrm{D}$ from JNT University, Kakinada, India in Computer 
Science. Her research interests include Image processing, Pattern Recognition and Database Management Systems. She is a life member of ISTE. At present she is doing her research at Srinivasa Ramanujan Research Forum-GIET, Rajahmundry.

Dr V Venkata Krishna received the B.Tech. (ECE) degree from Sri Venkateswara University. He completed his M. Tech. (Computer Science) from JNT University. He received his Ph.D in Computer Science from JNT University in 2004. He worked as Professor and Head for ten years in Mahatma Gandhi Institute of Technology, Hyderabad. After that he worked as Principal for Vidya Vikas College of Engineering, Hyderaba, Chaitanya Institute of Science \& Technology, JNTU, Kakinada and at present he is working as Principal in Chaitanya Institute of Engineering and Technology, JNTU, Rajahmundry, India. He is an advisory member for many Engineering colleges. He has published 25 research articles. Presently he is guiding 10 research scholars. He is a life member of ISTE and CSI. He is a member of Srinivasa Ramanujan Research Forum-GIET.

J. Sasi Kiran completed his B.Tech (EIE) Electronics \& Instrumentation Engineering in 2002 from J.N.T.U and M.Tech (C\&C) Computer \& Communication Engineering in 2005 from Bharath Institute of Higher Education \& research, Chennai and pursuing Ph.D (C.S.E) from Sathyabama University, Chennai. He worked as an Assistant Professor in Narayana Engg College, Nellore in C.S.E Dept. from July 2002 to June 2003 and worked as an Assistant Professor in Bharath Engg College, Chennai in C.S.E Dept. from July 2004 to May 2005. Presently he is working as an Associate Professor \& HOD CSE/IT in Vidya Vikas Institute of Technology, Chevella, Hyderabad, from June 2005 to till date. He has published research papers in various National, International conferences, proceedings and Journals. 